\title{
ABSTRACT
}

Spectral lines of the $\Delta V=2$ rotation vibration bands of $C O$ are well suited for study of photospheric motions and the mass ejection process in cool stars. We have obtained high spectral resolution $(1.8 \mathrm{~km} / \mathrm{sec})$ and high signal-to-noise $\left(>10^{2}\right)$ line profiles for a selection of $K$ and $\mathrm{M}$ giants. These profiles are being studied for evidence of gas motions in the photosphere and near circumstellar regions.

\section{THE OBSERVING PROGRAM}

During the last decade the observational possibilities for high resolution IR spectroscopy have progressed rapidly. It is now possible to acquire full resolved, high signal-to-noise spectra of a reasonable selection of bright giants. During winter 1978-1979 coordinated observing programs were initiated with a Fourier Transform Spectrometer (FTS) at the KPNO $4 \mathrm{~m}$ telescope. One program (D. N. B. Hall and K. Hinkle) was directed to precision measurement of radial velocities; the other program (L. Ramsey and S. T. Ridgway) was for study of photospheric temperature profiles and preparation of a spectral atlas. Observational material for these two programs was obtained joint 1 . The selected resolution was $\nu / \Delta \nu=160000$ (or $1.8 \mathrm{~km} / \mathrm{sec}$ at $4000 \mathrm{~cm}^{-1}$ ) and the spectral range 4000-6600 $\mathrm{cm}^{-1}$. The spectra were obtained in the daytime, since nighttime offers no advantage for such observations. Several studies based on these data will be published as they reach completion. Here we report on a preliminary examination of line profiles.

In line profile measurements it is naturally important to understand the intrinsic instrumental effects. For the FTS the theoretical line broadening at this modest resolution is negligible, and no line

* Operated by the Association of Universities for Research in Astronomy Inc., under contract with the National Science Foundation. 
asymmetry should be introduced by the instrument. For verification, we have examined the terrestrial $\mathrm{CO}_{2}$ band $2 v_{1}+2 v_{2}+v_{3}$ near $6348 \mathrm{~cm}^{-1}$ in one of the reference solar spectra. These lines are substantially narrower than the stellar lines, and hence a sufficient confirmation of instrument performance. No significant instrumental effects were detected.

The stellar line profiles discussed here are for a range of spectral types from KO to M4. In Table 1 the stars are listed with relevant parameters. Spectral types are from Wing (1978) when available

TABLE 1

List of Stars Observed

\begin{tabular}{|c|c|c|c|c|c|}
\hline$\underline{\mathrm{BS}}$ & Star & Spectral Type & BS & Star & Spectral Type \\
\hline 2990 & $\beta$ Gem & KO III & 1457 & $\alpha \mathrm{Tau}$ & $\mathrm{K} 5.7 \mathrm{III} \mathrm{AB}$ \\
\hline 5340 & $\alpha$ Boo & $\mathrm{K} 2$ III $\mathrm{p}$ & 337 & $\beta$ And & MO.5III A \\
\hline 3748 & $\alpha$ Hya & K4 III & 2286 & $\mu$ Gem & M3.3 III $A B$ \\
\hline 6705 & y Dra & $\mathrm{K} 5.0 \mathrm{III}$ & 7139 & $\delta^{2}$ Lyr & $\mathrm{M4} .3 \mathrm{II}$ \\
\hline
\end{tabular}

(decimal spectra types) or Hoffleit (1964) (integer spectral types). Luminosity classes are from the latter source.

\section{THE LINE PROFILES}

The complete data base is too extensive for reproduction here. Instead one example is shown in condensed form. In Figure 1 the profiles for low excitation CO Iines in $\mu$ Gem are shown superimposed. The line strengths are similar. This is in part because the decreasing population of the lower state for increasing rotational quantum $\mathrm{J}$ is partially offset by the increase in the statistical weight of higher J levels. Several features may be noted in Figure 1. The pseudo-emission peak due to the solar CO line is seen at $-25 \mathrm{~km} / \mathrm{sec}$. Blends with high $\mathrm{J}$ stellar CO lines are seen at positive velocities. Inconsistencies between the superimposed profiles may be attributed to observational noise and blends with weak lines. The profile bisectors show some scatter but in addition a systematic red shift in the line wings and a blue shift in the core. The reality of this effect may be confidently ascertained by examination of individual bisectors.

In general the profile bisectors show a red shift in the wings for both high and low excitation lines. Low excitation lines of the cooler stars show a blue shifted core. High excitation lines have a varying shift relative to low excitation lines. This information is summarized quantitatively in Figure 2 where the various shifts are plotted against spectral type. The core and wing shifts are relative to the halfintensity point; the high-low excitation shift is measured at halfintensity points. 


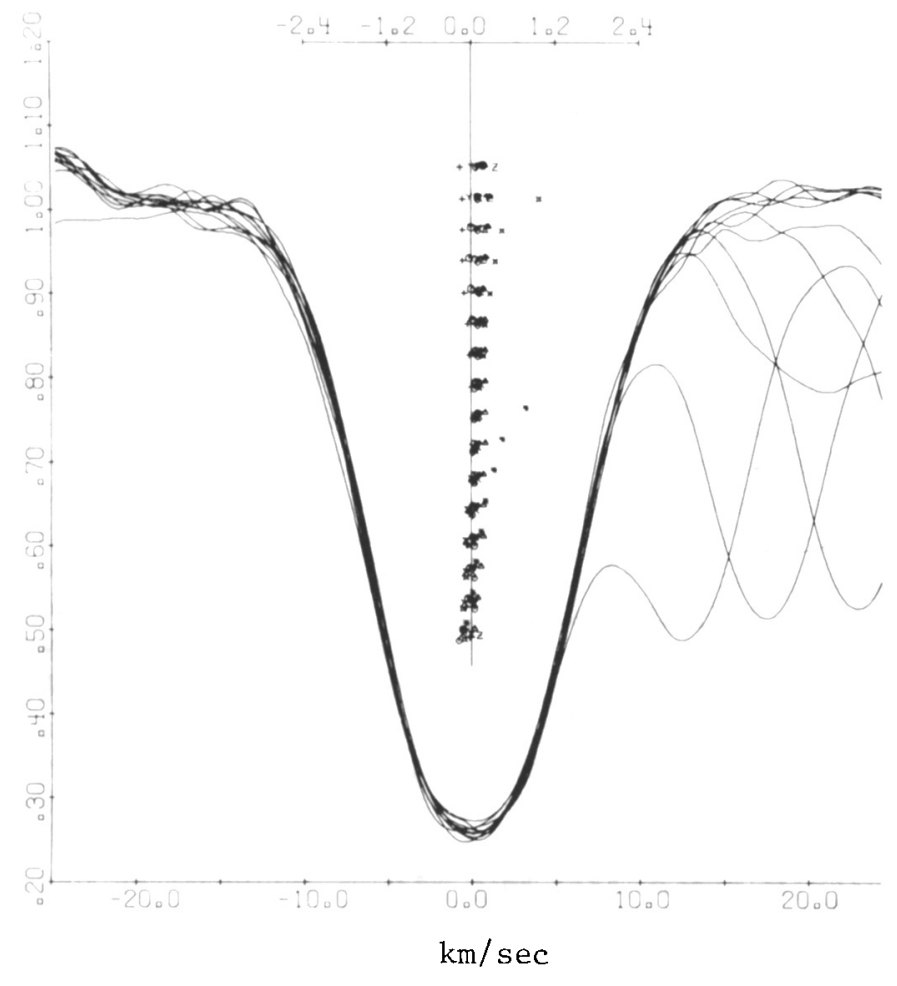

Fig. 1. The low excitation co lines of $\mu \mathrm{Gem}$.

As is well known, it is not possible to uniquely invert line profiles to derive mass motion, even for much superior data. Therefore, we will proceed by analogy with solar studies. Solar line profile bisectors show a characteristic $C$ shape, with red shifted wings and core. A spectral synthesis study incorporating detailed convective motions has been highly successful in explaning the appearance of the bisector plots (Dravins et al. 1980). The shift in the wings is due to the superposition of a blue shifted (ascending) component with a red shifted (descending) component. The descending component originates in a smaller surface area, and the spatial imbalance produces an asymmetric line. The "red shifted" core is actually due to material higher in the atmosphere that does not participate in the convective motion. By analogy, we suggest the following interpretation in cool stars. The wing shift is again due to convective motion. The blue shifted core, however, is to be associated with mass loss. The following discussion of the results rests on this interpretation. 


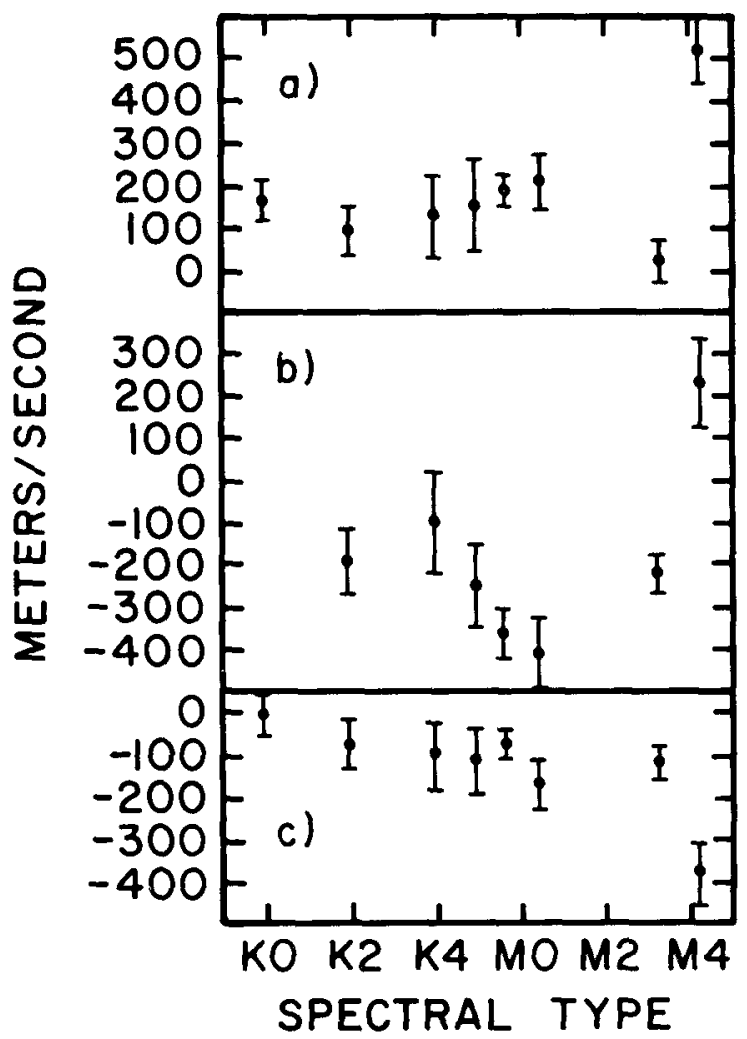

Fig. 2. Summary of bisector characteristics. a) Velocity of wing bisector relative to line half-intensity bisector (positive velocity signifies red shift). b) Velocity of high excitation lines relative to low excitation lines (measured at half-intensity bisectors). c) Line core velocity relative to halfintensity bisectors.

\section{IMPLICATION FOR CONVECTION}

The low excitation line wing bisector shift is plotted in Figure 2a. The results are consistent with a constant or gradually increasing shift for spectral types KO-MO, with a magnitude of $\sim 200 \mathrm{~m} / \mathrm{sec}$. Two general approaches may be used to estimate the implications for convective velocities.

From an anlysis of solar line shapes and related modeling of convection, Nordlund (1980) concludes that solar convective velocities are $\sim 1.5 \mathrm{~km} / \mathrm{sec}$ (vertical) and $2.5 \mathrm{~km} / \mathrm{sec}$ (horizontal). In disk center spectra, the shift of line wing bisector to half-intensity bisector is $\sim 50 \mathrm{~m} / \mathrm{sec}$ for strong, low excitation lines. In the solar case the integrated disk flux spectrum shows a substantially reduced line asymmetry. However, it is not obvious that this will be the case in cool giants, so no correction is made for that effect. Since the shifis shown in Figure $2 a$ are $\sim 4$ times the solar shifts, it appears qualitatively that a higher convective velocity, perhaps $\sim 4$ times higher, may be indicated in the late type giants. A second approach is to take 
note of the fact, mentioned earlier, that the wing asymmetry originates in spatial asymmetry. We make the extreme assumption that the line shape is due to the sum of two spatial components. Then assuming an unblended line shape, we experiment in summing spectra with varying line shift and relative line depth. In order to reproduce the observed bisector shift of $200 \mathrm{~m} / \mathrm{sec}$, a minimum velocity separation of the two components is found to be $\sim 6 \mathrm{~km} / \mathrm{sec}$. Presumably a more realistic integral over a continuous range of shifts would tend to reduce the asymmetry, so the $6 \mathrm{~km} / \mathrm{sec}$ value appears to be a lower limit. Coincidentally, it is consistent with the simple scaling from the solar case.

The relative blue shift of high excitation lines relative to low excitation lines is illustrated in Figure 2b. For the stars KO-M3, the correlation with Figure $2 \mathrm{a}$ is as expected and as observed in the high excitation solar lines: blue shift of the line increasing with excitation, and relative red asymmetry in the wings also increasing. The two coolest stars, $\mu$ Gem and $\delta^{2}$ Lyr, deviate from a simple extrapolation of the results for the hotter stars. The large wing shift in $\delta^{2}$ Lyr, according to the earlier discussion, would imply a convective velocity $\sim 10+\mathrm{km} / \mathrm{sec}$. This large value may be difficult to explain. Perhaps it should not be surprising, however, as similar values for macroturbulence have often been associated with late type high luminosity stars.

There can be no doubt of the reality of the large discrepancy between the two coolest stars, as both have very broad, well resolved, high $\mathrm{S} / \mathrm{N}$ profiles. One possible explanation would require intrinsic differences between the stars. Alternatively, variability of each star may contribute significantly. According to the suggestion of Schwarzschild (1975) convective patterns on the cool giants may be so large that only a few cells dominate the visible hemisphere. Variations in number and configuration across the disk could produce substantial changes in line profiles over a rotation. This possibility certainly deserves investigation by monitoring of time variations.

A related explanation could possibly account for the anamolous red shift of the $\delta^{2}$ Lyr high excitation lines (different not only in amplitude but in sign from the simple expectation). In a very extended atmosphere dominated by a few convective cells, the simple ideas which hold for spherical stars might break down. An elaborate model in three dimensions could be required.

\section{MASS LOSS}

The association of mass loss with blue shifts in the line core is a familiar suggestion, and in particular, line cores in the Co $\Delta v=1-0$ band revealed positive detection of circumstellar material in many cases (Hall et al. 1980). The $\Delta v=2-0$ band studied here is $\sim 50$ times weaker 
than the 1-0, but in some cases detection of circumstellar material should be possible here also. The relative blue shift of line cores is shown in Figure $2 \mathrm{c}$.

Only in the case of $\delta^{2}$ Lyr is the blue component of the absorption readily detectable by inspection of the profile. By reversing the line profile and differencing, the blue shifted component may be examined. The mean profile shows a blue shifted absorption at $\sim 2.5 \mathrm{~km} / \mathrm{sec}$ from line center, with a width $\sim 3 \mathrm{~km} / \mathrm{sec}$. The equivalent width is $0.035 \pm$ $0.01 \mathrm{~cm}^{-1}$. This implies a relatively large column abundance. If we assume the material is at a distance of 3 stellar radii, with $\mathrm{T}_{\text {exc }}$ $=2000 \mathrm{~K}$, the implied mass loss rate is $\sim 4 \times 10^{-7} \mathrm{MO} / \mathrm{yr}$. (At greater distance from the star, the rate would be higher as $\sim r^{2}$.) For comparison, Sanner (1976) derives a mass loss rate of $4 \times 10^{-8} \mathrm{MO} / \mathrm{yr}$. These numbers are probably consistent within the substantial uncertainties. It definitely appears that the material is in the near circumstellar region.

\section{FUTURE DEVELOPMENTS}

The data discussed here are the result of the first cool star infrared line profile study. These results required substantial amounts of $4 \mathrm{~m}$ telescope time - typically 4-6 hours per spectrum. On the other hand, the work was done in daytime, as appropriate for bright star astronomy. Substantial improvements may be foreseen. Simple restriction of the optical bandwidth to the $\mathrm{CO}$ band region would reduce the observing time by a factor of 30 . This gain could be allocated to an increase in resolution, but the resolution appears to be sufficient. If integration times $\sim 1$ hour were used, signa1-to-noise ratios $\sim 700$ would be achieved. Temporal monitoring would then also be a realistic possibility. Progress in those directions may be expected in the immediate future.

\section{REFERENCES}

Dravins, D., Lindegren, L. and Nordlund, A.: 1980, submitted to Astronomy and Astrophysics.

Hal1, D. N. B., Bernat, A. P., Hinkle, K. H. and Ridgway, S. T.: 1980, in press.

Hoffleit, D.: 1964, Catalogue of Bright Stars (Yale University Observatory, New Haven, Conn.).

Nordlund, A.: 1980, Stellar Turbulence, IAU Colloquium No. 51.

Sanner, F.: 1976, Ap. J. Supp 1.32 115.

Schwarzschi1d, M.: 1975, Ap. J. 195, 137.

Wing, R. F.: 1978, private communication. 